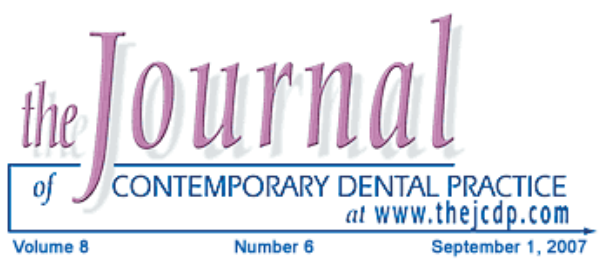

\title{
Total Impaction of Deciduous Maxillary Molars: Two Case Reports
}
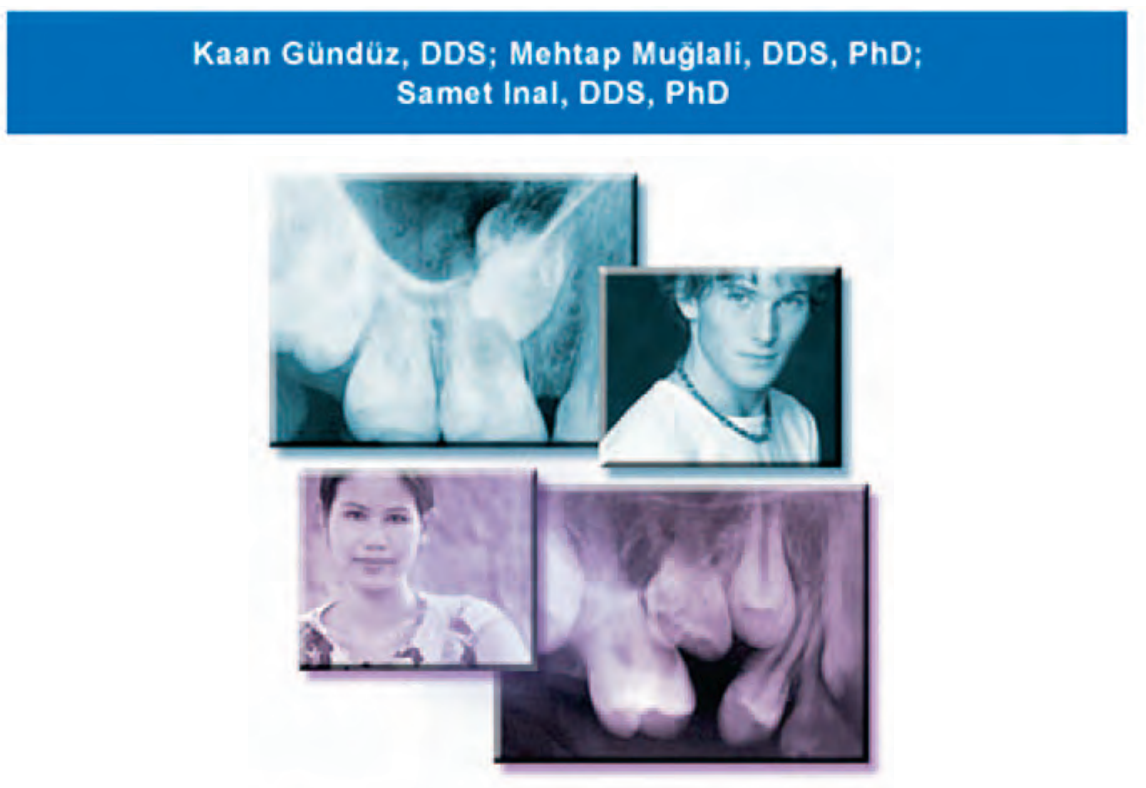

\section{Abstract}

Aim: The purpose of this report is to present two cases of totally impacted maxillary deciduous molars, considered a rarity in dental practice.

Background: Primary tooth impaction is quite rare during the development of primary dentition. Various factors contribute to the impaction of a deciduous tooth, including anklyosis, congenitally missing permanent teeth, defects in the periodontal membrane, trauma, injury of the periodontal ligament, precocious eruption of the first permanent molar, defective eruptive force, or a combination of these factors.

Reports: Case \#1: An 18-year-old male presented with a complaint of spontaneous repetitious pain in the maxillary right premolar region. The maxillary right second premolar was clinically absent. Panoramic and periapical radiographs revealed an impacted second premolar close to the inferior wall of the maxillary sinus and an impacted deciduous molar deeply embedded in bone within the maxillary sinus.

Case \#2: A 14-year-old girl presented with a complaint of crowding of the maxillary teeth. The maxillary right second premolar and the maxillary permanent canines were clinically absent. A panoramic radiograph revealed an impacted maxillary right second premolar and an impacted deciduous molar embedded within bone close to the inferior wall of the maxillary sinus.

(C) Seer Publishing 
Summary: The total impactation of deciduous teeth is a rare condition, and few cases have been reported in the literature. The condition generally affects the mandibular second deciduous molar and the maxillary first deciduous least often. In this paper, two cases of totally impacted maxillary deciduous molars are reported.

Keywords: Deciduous teeth, impaction

Citation: Gündüz K, Muğlali M, Inal S. Total Impaction of Deciduous Maxillary Molars: Two Case Reports. J Contemp Dent Pract 2007 September; (8)6:064-071.

\section{Introduction}

Submersion of a tooth occurs when a previously erupted tooth becomes embedded in the oral tissues. ${ }^{1}$ Other terms used in the literature to describe submergence are secondary retention, half retention, reinclusion, and ankylosis. ${ }^{2} \mathrm{~A}$ tooth can be truly impacted, but it is more common to find it has previously been present in the oral cavity. A tooth is considered submerged if its intact marginal ridges are more than 0.5 $\mathrm{mm}$ below the intact marginal ridges of the adjacent normal teeth. ${ }^{2}$ Secondary retention is a cessation of eruption of a tooth after initial emergence without a physical barrier in its path or is an ectopic position of the tooth. A tooth with secondary retention gradually appears more frequently in an infraposition, resulting in some cases being completely covered by the gingiva. ${ }^{2}$ The affected teeth are usually deciduous molars with the condition affecting the mandibular second molar most often and the maxillary first molar least often. ${ }^{1-5}$ The prevalence of children with submerged deciduous molars varies between different epidemiologic surveys ranging from 1.3 to $35 \%{ }^{3}$

The etiology of this condition is still unknown. ${ }^{1-6}$ However, recent histological and SEM studies of the root surfaces of extracted secondary teeth have shown most of these teeth to be anklyosed. ${ }^{2}$ The cause of this ankylosis is uncertain. ${ }^{1-3}$ The only factor found in a few cases is genetics.

Genuine reimpaction is a rare condition and results from a progessive loss of occlusal contact with no further growth in the height of the alveolar process of the submerged deciduous tooth. By contrast, the adjacent permanent teeth erupt; the regional alveolar processes move occlusally, and the submerged tooth is completely buried in the oral tissues. ${ }^{4}$
The purpose of this report is to present two cases of totally impacted maxillary deciduous molars, considered a rarity in dental practice.

\section{Case Reports}

\section{Case \#1}

An 18-year-old male presented with a complaint of spontaneous and repetitive pain in the maxillary right premolar region. The family's medical history was non-contributory. The maxillary right second premolar was clinically absent. Panoramic and periapical radiographs revealed an impacted second premolar close to the inferior wall of the maxillary sinus and an impacted deciduous molar deeply embedded within bone in the maxillary sinus (Figures 1 and 2). The impacted tooth showed external resorption.

The patient was scheduled for removal of the impacted second premolar and impacted deciduous tooth under local anesthesia. During surgery the impacted deciduous tooth was found to be ankylosed. The impacted teeth were carefully removed (Figure 3 ), however, during the procedure a minor communication with the maxillary sinus was noticed. The wound was closed with 3-0 silk suture material and the surgical procedure was completed. There were no complications following surgery.

\section{Case \#2}

A 14-year-old girl presented with a complaint of crowded maxillary teeth. The family's medical history was non-contributory. The intraoral examination revealed a Class II molar relationship and the absence of the maxillary right second premolar and the maxillary permanent canines. A panoramic radiograph revealed an impacted 


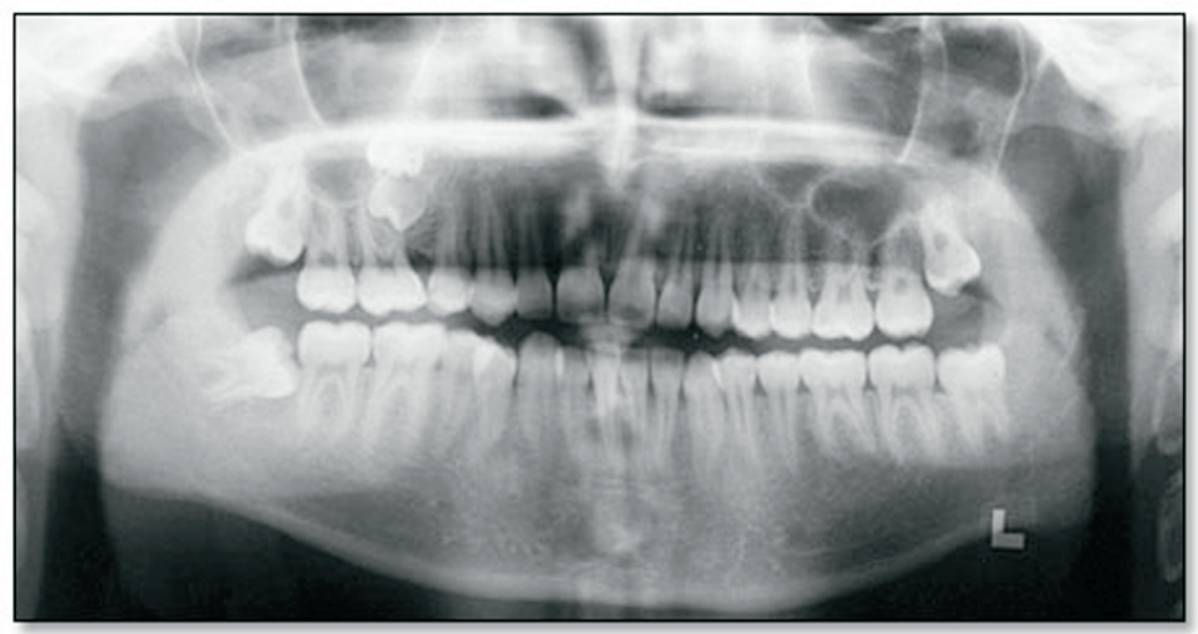

Figure 1. Panoramic radiograph showing a totally impacted deciduous maxillary right molar and impacted maxillary second premolar.

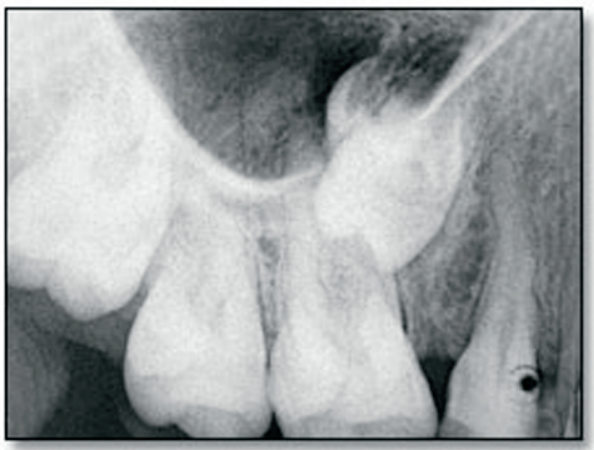

Figure 2. Periapical radiograph of the totally impacted deciduous tooth.

maxillary right second premolar and an impacted deciduous molar embedded within bone close to the inferior wall of the maxillary sinus (Figure 4). The impacted tooth was damaged due to caries and external resorption (Figure 5).

Surgical removal of the impacted tooth was carried out under local anesthesia without complications. An orthodontic eyelet with gold chain was bonded to the premolar at the time of surgery. A simple removable appliance was then used to commence traction of the impacted premolar using an elastomeric chain attached to the gold chain. After three months, the removable appliance was replaced by a conventional fixed orthodontic appliance. Alignment of the impacted premolar was completed without complication.

\section{Discussion}

The secondary retention of a tooth is a rare phenomenon caused by disturbances of the

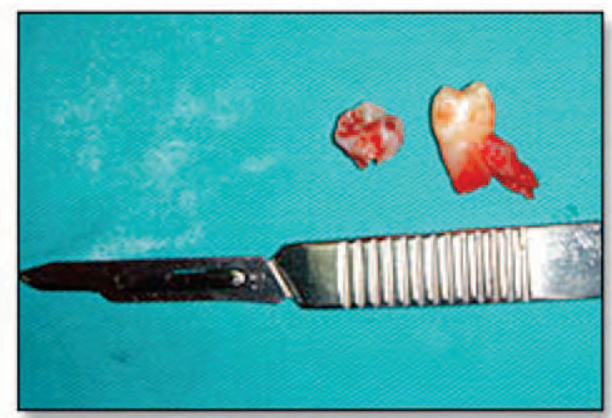

Figure 3. The totally impacted deciduous molar and impacted premolar after extraction.

physiological process of eruption. The presence of caries on the crown or even adjacent to restorations as well as the existence of abrasion facets indicates these teeth have previously erupted. ${ }^{2}$ Submerged teeth consist of various types from mild to severe and may involve from one to several teeth. Depending on the degree of submergence, the occlusion and position of the tooth germ may be affected. ${ }^{4}$ Total reimpaction of a tooth is considered a very rare phenomenon with very few cases presented in the literature. ${ }^{1-4}$ Two cases of totally impacted maxillary deciduous molars are presented in this paper.

Suggested factors possibly involved in submersion of deciduous teeth are anklyosis, congenitally missing permanent teeth, defects in the periodontal membrane, trauma, injury of the periodontal ligament, precocious eruption of the first permanent molar, defective eruptive force, or a combination of these factors. ${ }^{2-5}$ From all of 


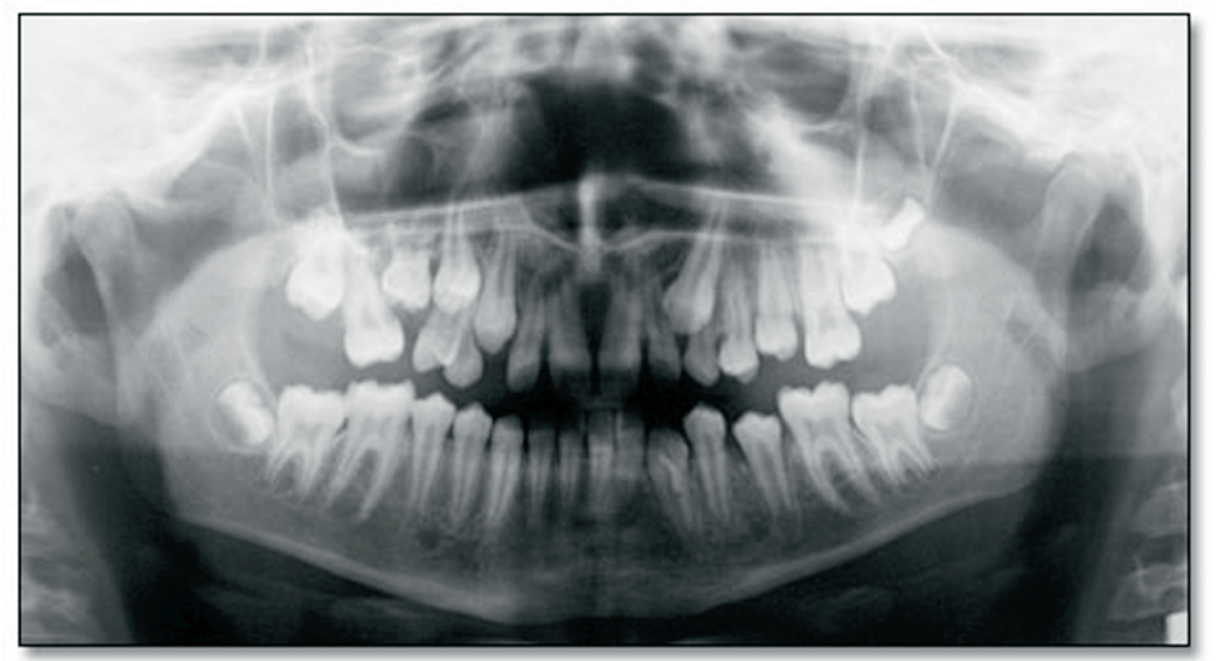

Figure 4. Panoramic radiograph showing a totally impacted deciduous maxillary right molar.

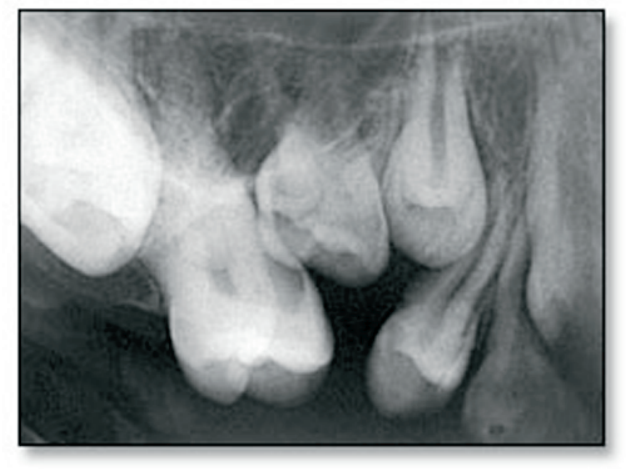

Figure 5. Periapical radiograph of the totally impacted deciduous tooth.

the cited etiological factors, ankylosis seems to be involved in the majority of cases or at least it is a coexisting factor and may explain why it is considered synonymous with the description of submersion. ${ }^{2}$ In the reported cases both of the impacted teeth were ankylosed.

Submerged teeth have a high potential to cause malocclusion by inclination of proximal teeth or extrusion of antagonistic teeth. ${ }^{6}$ Rygh et al., ${ }^{6}$
Ten Cate, ${ }^{7}$ Thornton et al., ${ }^{8}$ and Biederman ${ }^{9}$ all recommended early tooth extraction for the treatment of submerged teeth. On the contrary, Mcdonald et al. ${ }^{10}$ insisted if the cooperation of patients is obtained, observation is the best treatment since some submerged teeth exfoliate while the rest require extraction. In the first presented case the impacted second premolar and deciduous tooth were removed because of the severe infraclusion. In the second case the preferable treatment option was extraction because the submerged deciduous right maxillary second molar probably inhibited movement of the tooth germ of the right maxillary second premolar, forcing it into a malposition.

\section{Summary}

The total impactation of deciduous teeth is a rare condition, and few cases have been reported in the literature. The condition generally affects the mandibular second deciduous molar and the maxillary first deciduous least often. In this paper two cases of totally impacted maxillary deciduous molars are reported. 


\section{References}

1. Allsopp P, Johns M. Restored, totally submerged deciduous molar. Case report. Aust Dent J. 1982 Feb; 27(1): 27-9.

2. Antoniades K, Kavadia S, Milioti K, Antoniades V, Markovitsi E. Submerged teeth. J Clin Pediatr Dent. 2002 Spring; 26(3): 239-42.

3. Steigman S, Koyoumdjisky-Kaye E, Matrai Y. Relationship of submerged deciduous molars to root resorption and development of permanent successors. J Dent Res. 1974 Jan-Feb; 53(1): 88-93.

4. Miyanaga M, Takei K, Maeda T. Observation of a child with multiple submerged primary teeth. ASDC J Dent Child. 1998 Nov-Dec; 65(6): 495-8.

5. Rune B, Sarnas KV. Root resorption and submergence in retained deciduous second molars. A mixed-longitudinal study of 77 children with developmental absence of second premolars. Eur $\mathrm{J}$ Orthod. 1984 May; 6(2): 123-31.

6. Rygh P, Reitan K. Changes in the supporting tissues of submerged deciduous molars with and without permanent successors. Odont T. 1964 72: 345-62.

7. Ten Cate. Orbans oral histology and embryology, 7th ed. St Louis, Mosby Co, 1972, p329.

8. Thornton M, Zimmerman E. Ankylosis of primary teeth. J Dent Child. 1964 31: 120-126.

9. Biederman W. The ankylosed tooth. Dent Clin N Am. 1964 493-508.

10. McDonald RE, Avery DR, Jeffrey A. Dentistry for the Child and Adolescent, 8th ed. St.Louis. Mosby Co, 1983, p116-21.

\section{About the Authors}

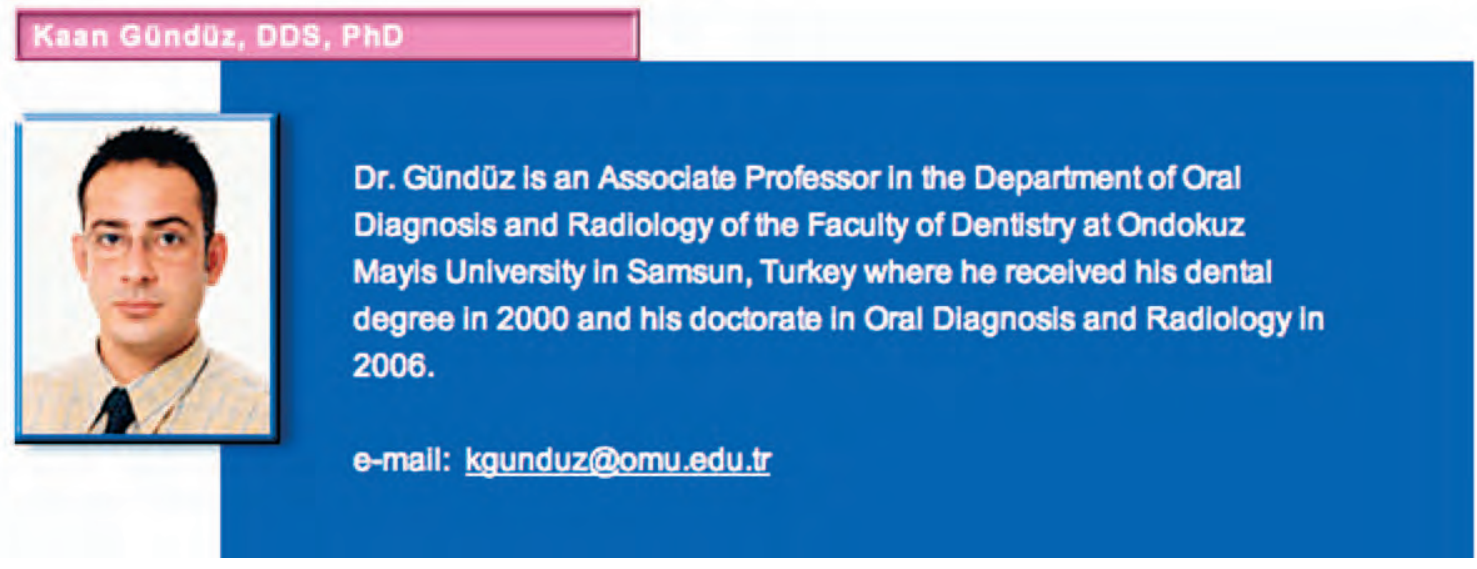

\section{Mohtap Muglall, DDS, PhD}

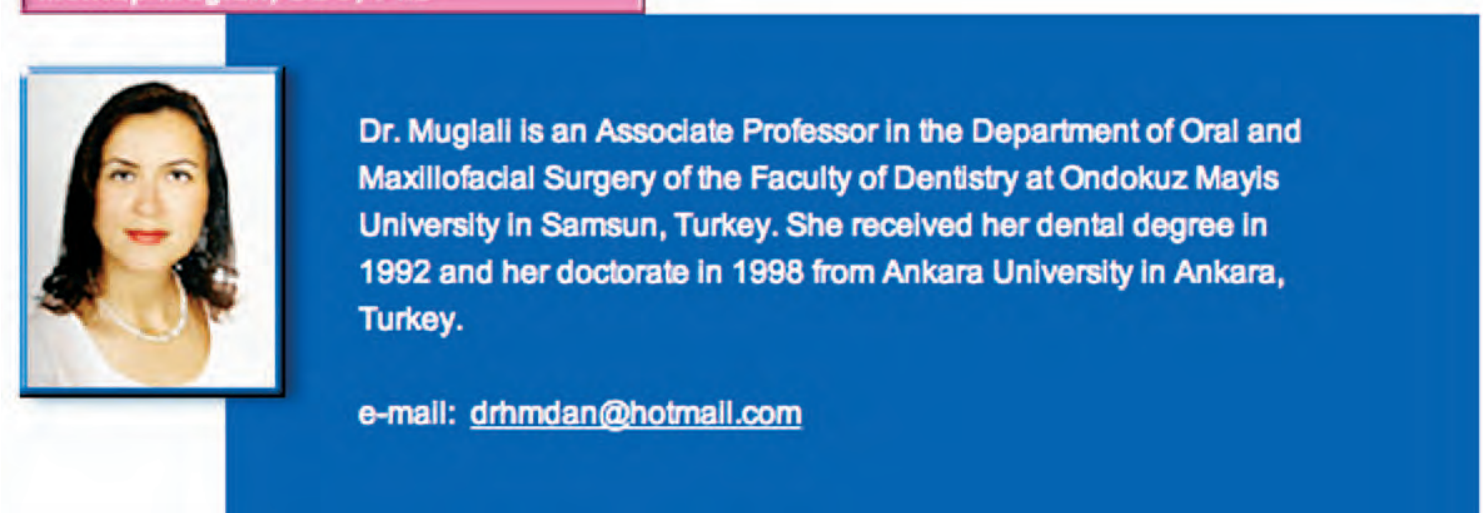




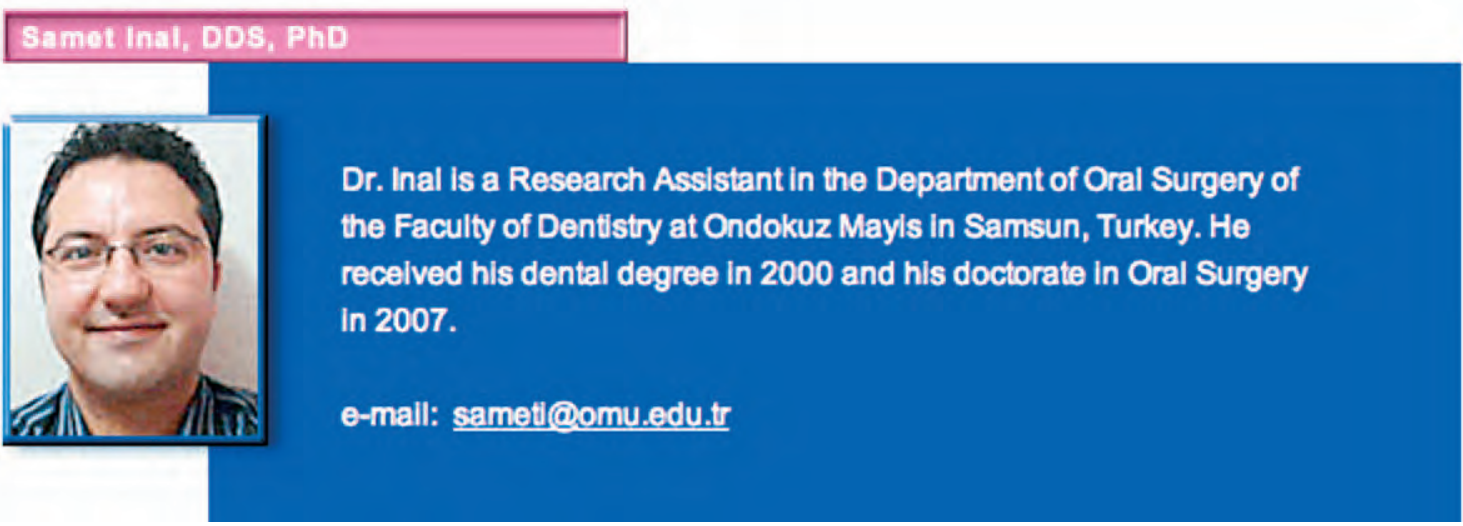

